\title{
Atenção primária à saúde de indígenas sul-americanos: revisão integrativa da literatura
}

\author{
Luiza Fernandes Fonseca Sandes, ${ }^{1}$ Daniel Antunes Freitas, ${ }^{2}$ Maria \\ Fernanda Neves Silveira de Souza ${ }^{1}$ e Kellen Bruna de Sousa Leite ${ }^{1}$
}

Como citar Sandes LFF, Freitas DA, Souza MFNS, Leite KBS. Atenção primária à saúde de indígenas sul-americanos: revisão integrativa da literatura. Rev Panam Salud Publica. 2018;42:e163. https://doi.org/10.26633/ RPSP.2018.163

RESUMO Objetivo. Revisar a literatura acerca do acesso à atenção primária à saúde (APS) por comunidades indígenas da América do Sul, identificando os principais obstáculos a esse acesso.

Métodos. Revisão integrativa de artigos publicados de 2007 a 2017 nas bases de dados LILACS, PubMed e SciELO. Para a busca, foram utilizados os descritores "indígenas AND saúde AND Brasil" e "indígenas AND saúde NOT Brasil" nos idiomas português e inglês. Foram incluídos artigos publicados em inglês, português ou espanhol e que abordassem estritamente a APS em indígenas sul-americanos.

Resultados. Foram incluídos 40 artigos que descreveram aspectos da APS de indígenas em oito países: Brasil, Peru, Colômbia, Bolívia, Argentina, Chile, Paraguai e Equador. Os principais obstáculos de acesso à APS detectados foram a dificuldade de acesso aos serviços de saúde mais próximos das aldeias; linguagem e ilustraçães das cartilhas de educação em saúde inapropriadas ao contexto indígena; dificuldade de comunicação com os profissionais de saúde; carência de meios de transporte adequados até as unidades de saúde; escassez de dados epidemiológicos das aldeias indígenas; ausência de informação sobre as culturas indígenas locais; e medo de discriminação ou humilhação por parte do paciente indígena.

Conclusões. Ainda são escassos os estudos sobre saúde indígena na América do Sul. Também é evidente que os sistemas de saúde nacionais ainda precisam avançar na direção de uma medicina intercultural, de respeito às realidades sociais, culturais e econômicas de todas as comunidades assistidas, com conhecimento e consideração pelas diferentes formas de cuidado.

Palavras-chave População indígena; saúde de populações indígenas; atenção primária à saúde; América Latina.

Existem na América do Sul numerosos grupos indígenas, fragmentados em pequenas e abundantes áreas que se

\footnotetext{
Universidade Estadual de Montes Claros (Unimontes), Faculdade de Medicina, Montes Claros (MG), Brasil. Correspondência: Luiza Fernandes Fonseca Sandes, luizaffsandes@gmail.com

2 Departamento de Saúde Mental e Saúde Coletiva, Universidade Estadual de Montes Claros (Unimontes), Montes Claros (MG), Brasil.
}

estendem por todo o continente. Essas comunidades, embora culturalmente distintas entre si, apresentam indicadores de saúde semelhantes e sofrem a mesma marginalização por parte dos sistemas de saúde e discriminação por parte da população não indígena. $\mathrm{Na}$ última década, apesar das significativas melhorias nos indicadores de saúde em diversos países sul-americanos, com redução das taxas de mortalidade infantil e materna e da desnutrição crônica, essas melhorias não se refletiram plenamente nas populações indígenas, que não são devidamente contabilizadas nos censos (1).

Entre os países sul-americanos com maior número de tribos indígenas estão 
Peru, Bolívia e Equador (2). Em 2010, foi estimada a existência de, no mínimo, 826 grupos indígenas na América Latina, correspondendo a cerca de 45 milhões de pessoas (3), com mais de 1000 dialetos utilizados entre as tribos (2). Essas populações, com genética e epidemiologia diferentes dos seus colonizadores europeus, enfrentaram taxas de mortalidade catastróficas séculos atrás quando entraram em contato com novos patógenos, trazidos por espanhóis e portugueses. Atualmente, ainda é possível identificar os reflexos do choque entre indígenas e europeus através de suscetibilidades a determinadas doenças e epidemias que continuam ocorrendo dentro das aldeias (4).

A etnicidade é um importante fator determinante das condições de saúde de um povo, influenciando taxas de morbidade e mortalidade em diferentes grupos étnicos e interferindo no acesso aos serviços de saúde para determinados estratos populacionais (5). Dentro das populações indígenas, a autopercepção do adoecer e a relação das tribos com a prática biomédica, com medicamentos industrializados e com agentes de saúde externos é particular de cada grupo. Essas particularidades são complicadas pela dificuldade geográfica de acesso às aldeias, barreiras linguísticas e barreiras de informação, o que determina diferenças entre a saúde prestada para as populações urbanas não tradicionais e os povos indígenas.

No final da década de 1970, a conferência de Alma-Ata explicitou a urgência de desenvolver e promover uma saúde igualitária para todos os povos. A Declaração de Alma-Ata, de 1978, estabelece como meta a busca por uma saúde que englobe bem estar social, físico e mental completo, e não apenas a simples ausência de doenças (6). Essa declaração consolidou-se como uma das bases de referência para a Constituição Federal do Brasil, promulgada em 1988, e do Sistema Único de Saúde (SUS) brasileiro (7-9). Através da criação do SUS, baseado em princípios interculturais, com políticas de saúde específicas para populações indígenas, o Brasil tornou-se um dos países sul-americanos pioneiros nesse campo, influenciando os demais países do continente.

Durante as décadas de 1980 e 1990, na linha do que que se seguiu no Brasil, surgiram na América Latina diversos projetos para criação de sistemas universais de saúde. Entretanto, atualmente, vários desses sistemas na América do Sul não estabelecem acesso universal, segregando diversos grupos étnicos e minoritários, como indígenas e afrodescendentes (3).

No final do século $X X$, impelidos pelo intenso e vertiginoso processo de globalização, emergiram diversos movimentos igualitários nas Américas, com reivindicações culturais, sociais e de gênero, apoiados pela Organização Pan-Americana da Saúde (OPAS). Nesse sentido, a OPAS, a Organização Mundial da Saúde (OMS) e a Organização das Nações Unidas (ONU) propuseram e incentivaram a integração dos sistemas de saúde da América Latina, desenvolvidos de maneira independente por cada governo nacional, porém incorporando uma abordagem intercultural e propostas étnico-políticas com reconhecimento das identidades coletivas e dos direitos dos povos indígenas (10-12).

Considerando as políticas públicas propostas para estabelecer melhores condições para as populações indígenas da América do Sul, o objetivo deste trabalho foi detalhar a atenção primária à saúde (APS) desses povos. A partir de uma revisão integrativa da literatura, foram pormenorizados o panorama da saúde indígena e os principais obstáculos ainda enfrentados por pacientes indígenas no acesso à APS, em países sul-americanos, nos últimos 10 anos.

\section{MATERIAIS E MÉTODOS}

Foi realizada uma revisão integrativa da literatura a partir de pesquisa em bases de dados, desenvolvida a partir das seis etapas propostas por Mendes et al. (13): estabelecimento de hipótese ou questão de pesquisa; amostragem ou busca na literatura; categorização dos estudos; avaliação dos estudos incluídos na revisão; interpretação dos resultados; e síntese do conhecimento ou apresentação da revisão. Essas etapas foram seguidas a fim de garantir o rigor metodológico do estudo. As perguntas "qual é o panorama das condições de atenção à saúde dos povos indígenas em países sul-americanos?" e "quais os principais obstáculos ainda enfrentados pelos indígenas, relativos ao acesso à saúde primária?" foram as questões norteadoras definidas para a investigação.

A busca dos artigos foi realizada em dezembro de 2017 nas bases de dados LILACS (http://lilacs.bvsalud.org/), PubMed (https://www.ncbi.nlm.nih. gov/pubmed/) e SciELO (http:/ / www. scielo.org/php/index.php). As estratégias de busca utilizaram uma combinação de três descritores em ciências da saúde (DeCS) e medical subject headings (MeSH). A busca por estudos nas bases de dados LILACS e SciELO utilizou a seguinte estratégia: "indígenas AND saúde AND Brasil", "indígenas AND saúde NOT Brasil", "indígena AND salud AND Brasil", "indígena AND salud NOT Brasil", "indigenous AND health AND Brazil" e "indigenous AND health NOT Brazil". Já na base de dados PubMed, foram utilizados apenas os descritores combinados no idioma inglês. A busca de dados foi realizada por três pesquisadores, de maneira independente e sem interferências externas. Dessa maneira, após a seleção de amostragem individual de cada pesquisador, as três amostras de artigos foram agrupadas e os artigos duplicados foram excluídos.

A análise dos dados seguiu critérios de inclusão baseados no tema proposto pela presente pesquisa: estudos realizados nos últimos 10 anos, de 2007 a 2017; que possuíam texto integral disponível on-line, com acesso aberto gratuito; publicados nos idiomas inglês, português ou espanhol; e que abordassem as condições de atenção à saúde, bem como os obstáculos relativos ao acesso à APS pelos povos indígenas sul-americanos. Foram excluídos os estudos que tratavam da atenção secundária e terciária à saúde e que não se referiam a populações indígenas sul-americanas, artigos repetidos e trabalhos que não foram realizados nos últimos 10 anos.

A avaliação do nível de evidência dos artigos na amostra final foi realizada com base nas proposições de Melnyk e Fineout-Overholt (14): I - revisão sistemática ou meta-análise (maior nível de evidência); II - ensaio controlado aleatório; III - ensaio controlado sem aleatoriedade; IV - estudo de caso-controle ou estudo de coorte; $\mathrm{V}$ - revisão sistemática de estudo qualitativo ou descritivo; VI estudo qualitativo ou descritivo; VII - parecer ou consenso de expertises (menor nível de evidência).

\section{RESULTADOS}

A aplicação dos descritores resultou na localização de 5234 artigos nas três bases de dados on-line: 613 na base SciELO, 3371 na PubMed e 1250 na base LILACS. Após aplicação dos critérios de inclusão 
e exclusão previamente estabelecidos, foram selecionados 67 artigos para leitura na íntegra, dos quais 27 foram excluídos por duplicidade, totalizando 40 artigos $(1-5,10,15-48)$ na amostra final (figura 1).

Do total de 40 artigos, 11 foram em inglês, 20 em português e nove em espanhol. A tabela 1 resume os estudos que compõem essa revisão sistemática integrativa, categorizando os aspectos da saúde indígena abordados, o delineamento do estudo, o país de origem dos grupos indígenas e o idioma das publicações. Foram descritos aspectos da APS de indígenas em oito países da América do Sul, sendo o Brasil aquele com maior número de estudos $(\mathrm{n}=24)$, seguido por Peru (n = 9), Colômbia $(n=3)$, Bolívia $(n=3)$, Argentina $(n=3)$, Chile $(n=2)$, Paraguai $(n=2)$ e Equador $(\mathrm{n}=1)$.

\section{Brasil}

A população indígena brasileira corresponde a aproximadamente $817 \mathrm{mil}$ pessoas, agrupadas em 220 tribos distribuídas em 4774 aldeias, dentro de 438 municípios brasileiros, conforme dados de 2016 (26). O perfil epidemiológico desses povos é notadamente distinto daquele de comunidades não indígenas, com elevadas taxas de morbimortalidade, principalmente relacionadas a doenças infecto-parasitárias (27). Devido à transição epidemiológica enfrentada pelas tribos, resultado da aquisição de hábitos alimentares e sociais ocidentais, é possível verificar o surgimento de doenças previamente inexistentes nas aldeias, como hipertensão arterial, diabetes e câncer (27).

No Brasil, os movimentos em prol da saúde indígena se consolidaram na década de 1990, após o estabelecimento do SUS como sistema universal de saúde e o reconhecimento, na Constituição Federal de 1988, dos direitos indígenas a suas terras, cultura, línguas e tradições $(26,28)$. Nesse sentido, foi implantada a Política Nacional de Atenção à Saúde dos Povos Indígenas, em 1999, criando o Subsistema de Atenção à Saúde Indígena (SASI). O SASI tem como base os Distritos Sanitários Especiais Indígenas (DSEI), que devem oferecer APS nas terras indígenas com equipes multidisciplinares $(26,27,29)$. Contudo, a rede de subsistemas ainda não se encontra plenamente

FIGURA 1. Fluxograma de revisão sistemática integrativa sobre atenção primária à saúde de indígenas sul-americanos e seleção da amostra final de artigos

Identificação pelos
descritores
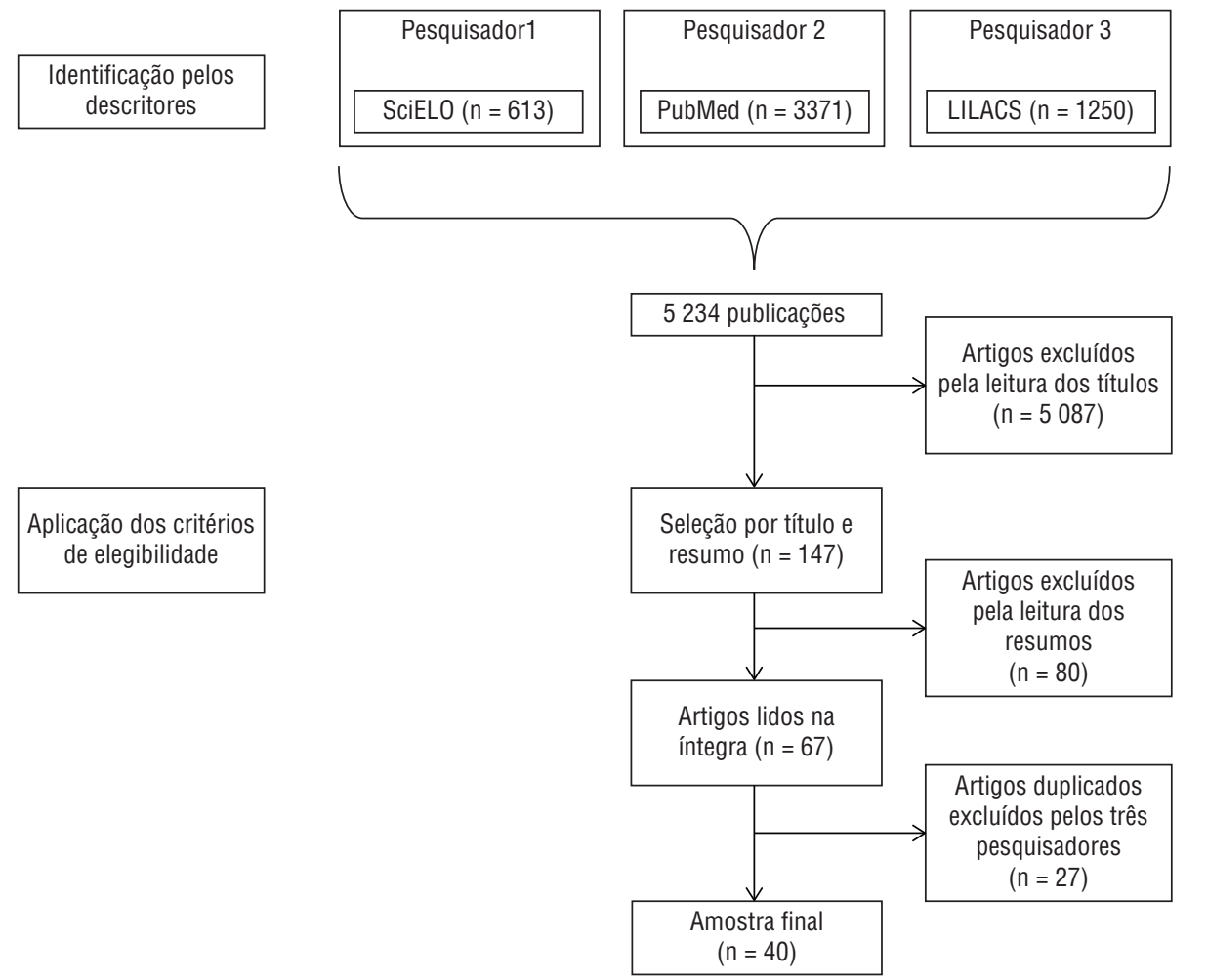

consolidada, já que, apesar da criação dos DSEI, a falta de infraestrutura física e de recursos humanos ainda é uma realidade, resultando em rotatividade de profissionais e consequente irregularidade na oferta dos serviços, devido à perda de profissionais com experiência em saúde indígena (30-32).

Desde 2011 existe grande incentivo governamental para o fortalecimento dos serviços de APS, através do programa Saúde da Família, que expandiu a cobertura do SUS para cidadãos em áreas remotas e distantes dos grandes centros (26). Em 2013, com a implantação do Programa Mais Médicos (PMM), mais de 18 mil médicos foram alocados em regiões consideradas de difícil acesso e maior vulnerabilidade, inclusive territórios indígenas; o PMM teve avaliações positivas por parte da população indígena assistida $(26,30,33)$. Problemas de efetivação desse projeto também foram verificados, como, por exemplo, a dificuldade de comunicação e o embate de culturas, cenário que seria suavizado se fossem oferecidos cursos aos profissionais para capacitação sobre a cultura e a língua dos povos nativos (30). Uma das estratégias desenvolvidas para enfrentar esse problema foi a institucionalização do agente indígena de saúde (AIS) como parte das equipes que prestam APS nas aldeias $(34,35)$. Os AIS têm o papel de atuar na promoção da saúde e prevenção das doenças, articulando práticas populares, tradicionais e biomédicas de atenção à saúde (34, 36-40).

A maioria das causas de morte entre crianças indígenas no Brasil ocorre por enfermidades preveníveis. Entre as doenças mais prevalentes estão tuberculose, malária, diarreia, parasitoses, desnutrição, dermatoses e doenças sexualmente transmissíveis (26). Nas últimas décadas houve expressiva redução das taxas de mortalidade infantil entre crianças brasileiras. No entanto, as taxas entre crianças indígenas têm decrescido em menor intensidade (26). De 2002 a 2007, a desnutrição infantil no país decresceu mais de $60 \%$; todavia, a prevalência de crianças com baixo peso é 4 vezes maior na região Norte, particularmente em associação às comunidades indígenas (26). A cobertura vacinal no Brasil sofreu grande ampliação, através de estratégias de imunização regulares, inclusive nas populações indígenas (26). 
TABELA 1. Categorização de todos os estudos utilizados na amostra final e analisados integralmente para composição da revisão sistemática integrativa sobre atenção primária à saúde de indígenas sul-americanos

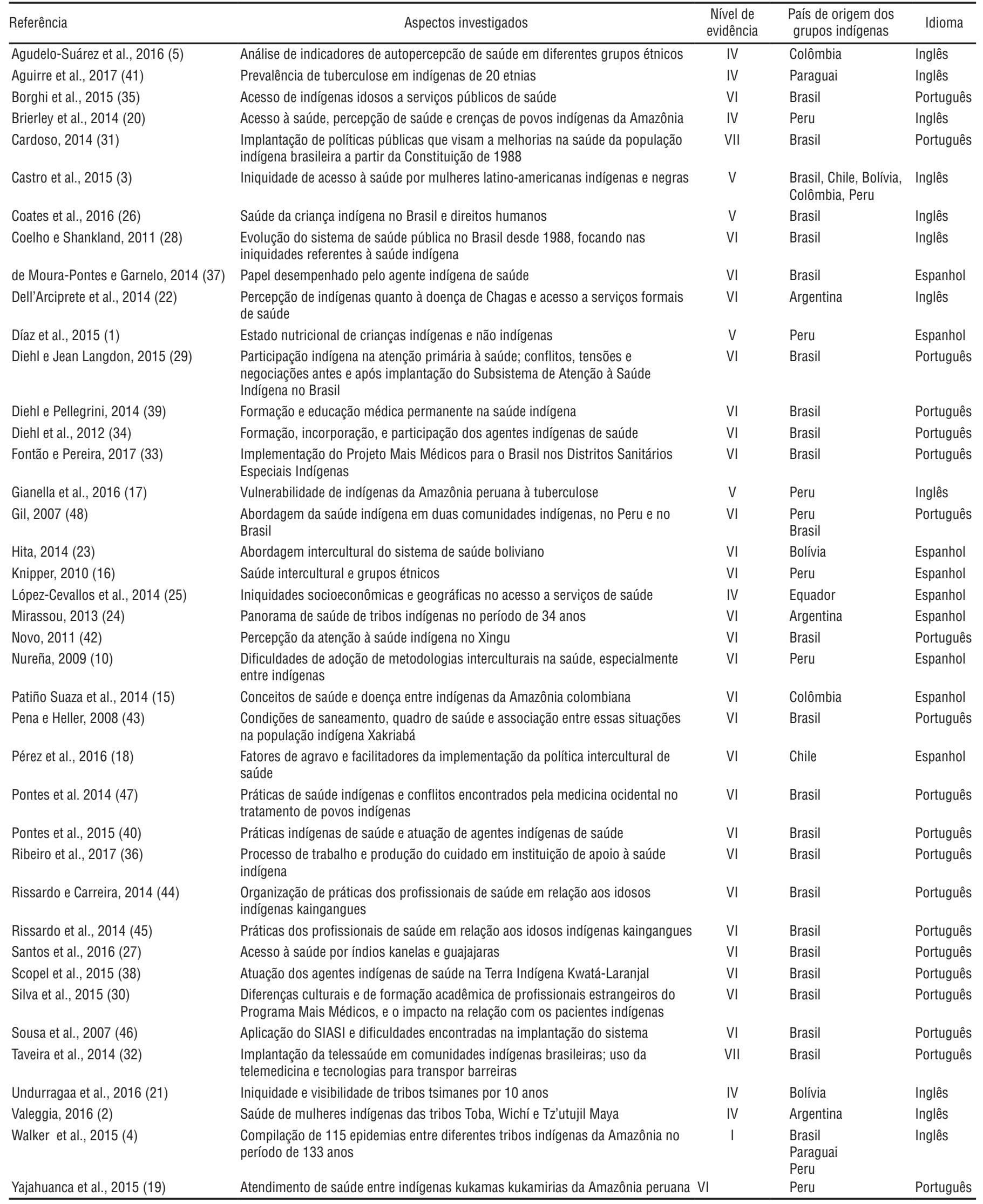




\section{Peru}

O Peru é um dos países da América do Sul com maior número de comunidades indígenas em seu território. Estima-se que cerca de um terço da população peruana seja indígena, vivendo majoritariamente na Amazônia peruana, região com maior diversidade de povos indígenas distintos entre si, com tradições seculares e saberes tradicionais específicos (19). De acordo com estatísticas oficiais, existem no Peru cerca de 72 grupos étnicos típicos. Essas populações indígenas enfrentam acentuadas taxas de mortalidade infantil e materna, subnutrição e doenças infecciosas (10).

O sistema de saúde peruano é atualmente voltado para um país homogêneo e não considera o rico multiculturalismo em seu território. Em 2002, o governo peruano implantou o Seguro Integral de Salud (SIS), que foi importante para a redução de taxas de mortalidade infantil, mas não assegurou acesso integral à saúde para todos (17). Em 2004, foram estabelecidas pelo governo as Estrategias Sanitarias Nacionales (ESN), que propunham políticas de saúde para povos indígenas (10). Entretanto, é importante salientar que, até 2014, não existia no Peru nenhum órgão governamental direcionado para gestão de políticas voltadas para populações indígenas (19).

Em 2014, o governo decretou que todos os povos indígenas da Amazônia peruana fossem reconhecidos como vivendo em extrema pobreza para que fossem assistidos pelo SIS, aumentando consideravelmente a cobertura do sistema de saúde. As autoridades ratificaram a necessidade de estratégias de saúde interculturais e garantiram o direito à saúde para os povos indígenas (17).

Os povos indígenas do Peru sobrevivem em áreas ricas em madeiras, minerais e óleos naturais, que atraem trabalhadores estrangeiros regularmente, levando novos patógenos para as aldeias (17). Entre as doenças com maiores incidências entre essas comunidades estão tuberculose, parasitoses, malária, subnutrição e surtos diarreicos agudos $(10,20)$. O acesso aos serviços básicos de saúde sofreu melhorias nas últimas décadas, mas continua deficiente. A cobertura vacinal e outras medidas sanitárias continuam sendo menores em crianças indígenas do que em crianças não indígenas. Além disso, 78\% das crianças indígenas peruanas vivem em situação de pobreza $(1,17)$. Um estudo conduzido em 2014 (20) com tribos indígenas peruanas constatou que $57 \%$ da população questionada não conseguiram assistência médica quando necessário, enquanto $72 \%$ não tinham esse acesso em 1999 (20). Práticas de medicina tradicional como curandeirismo e xamanismo continuam sendo largamente utilizadas por até $80 \%$ dos indígenas peruanos $(17,20)$. $\mathrm{O}$ atendimento de demandas de baixa complexidade é feito por clínicas em zonas semiurbanas e, raramente, dentro das aldeias, onde são realizadas atividades preventivas, diagnóstico precoce e tratamento oportuno de doenças da comunidade (29).

\section{Bolívia}

Povos indígenas na Bolívia, como os tsimanes, vivem no interior das florestas tropicais (21). Mulheres indígenas bolivianas apresentam elevadas taxas de morbidade durante a gravidez e frequentemente não procuram serviços de saúde, pois sentem-se excluídas e inseguras nesse ambiente (21). Entre as dificuldades de acesso à saúde relatadas nas aldeias bolivianas, os aymarás referem falta de comunicação adequada entre os profissionais de saúde e os pacientes indígenas (22).

O sistema de saúde boliviano foi estabelecido como universal em 2011, centrado na APS e na medicina social (23). Entretanto, esse sistema ainda não dá conta de todas as demandas populacionais da Bolívia e não garante a participação comunitária na formulação das políticas (23).

\section{Colômbia}

A Colômbia é rica em diversidade étnica e linguística, com um grupo étnico indígena minoritário que representa aproximadamente $14 \%$ da população do país (2). O direito à saúde dos povos indígenas não é assegurado pelo governo e, segundo informações do Ministério da Saúde, as taxas de mortalidade infantil e materna na Amazônia colombiana foram as mais altas do país (15).

Dentre os grupos étnicos colombianos, os indígenas apresentam maiores índices de doenças mentais (2). A medicina tradicional continua sendo amplamente utilizada e, inclusive, preferida pelos indígenas colombianos para a cura de enfermidades (15). Em pesquisa etnográfica realizada de 2010 a 2013 em tribos da Amazônia colombiana, o reconhecimento das práticas de cuidado tradicionais das tribos, através dos curandeiros, e o respeito pelos pacientes das aldeias por parte dos médicos estiveram entre as demandas destacadas pelas comunidades (15).

\section{Argentina}

Na Argentina a proporção de indígenas, entre os quais se incluem as tribos Mbya-Guarani e Toba, é expressivamente menor $(2,22)$. Algumas aldeias indígenas do país estão em fase de transição epidemiológica, convivendo com patologias seculares não erradicadas concomitantemente a situações emergentes relacionadas ao estilo de vida moderno, como obesidade, sedentarismo, diabetes e alcoolismo (24). Através de censos realizados pelo ministério da saúde argentino, foi possível verificar queda significativa das taxas de mortalidade infantil entre aborígenes após a efetivação de estratégias de APS no país (24). Ademais, nas últimas décadas, ocorreu redução da incidência de tuberculose em algumas aldeias após política ampla de imunização (24).

\section{Chile}

Uma das principais comunidades étnico-culturais do Chile é a dos mapuches, que representam $87 \%$ de todas as etnias reconhecidas pelo governo chileno em seu território (18). Os mapuches ainda enfrentam elevadas taxas de mortalidade infantil e alta prevalência de tuberculose.

Em 1996, o governo chileno implantou o Programa Especial de Salud y Pueblos Indígenas e, em 2006, formulou a Politica de Salud y Pueblos Indígenas. Essas iniciativas se basearam em um modelo intercultural de saúde e nos princípios de equidade e participação comunitária (18).

As aldeias indígenas chilenas possuem perfil epidemiológico único e em transição, com enfermidades típicas de regiões subdesenvolvidas coexistindo com outras associadas à modernidade (18). Uma das principais demandas dos profissionais de saúde chilenos para atendimento adequado dos mapuches é o acesso a informação e educação sobre a cultura e crenças desse povo (18).

\section{Paraguai}

O Paraguai possui aproximadamente 20 grupos étnicos, que correspondiam a 
cerca de $1,7 \%$ de toda a população paraguaia em 2012 (41). Nessas comunidades, 112800 pessoas pertencem a grupos indígenas e possuem altas taxas de prevalência de tuberculose. Os relatos sobre indígenas paraguaios indicam que essa população costumeiramente atrasa a busca por cuidado médico formal devido a uma relativa minimização dos sintomas, o que, consequentemente, causa atraso no diagnóstico e na cura de doenças (41).

\section{Equador}

O Equador é um dos países sul-americanos com menor cobertura pelo sistema de saúde nacional - aproximadamente $25 \%$ da população em 2014 (25). A pobreza afeta $40 \%$ dos cidadãos equatorianos, com maiores índices nas comunidades rurais e indígenas (25).

Análises estatísticas mostram que apenas $18 \%$ dos povos indígenas equatorianos possuem acesso a serviços curativos de saúde, apesar das mudanças expressivas no sistema de saúde nacional na última década. Através de incentivo de organizações indígenas como a Confederación de Nacionalidades Indígenas del Ecuador (CONAIE), o novo sistema de saúde equatoriano passou a integrar princípios interculturais, aliando medicina ocidental e tradicional, como ferramenta para reverter as precárias condições desses povos (25).

\section{DISCUSSÃO}

Apesar de serem habitantes seculares da América do Sul, apenas nos últimos 30 anos a saúde dos indígenas começou a ser tratada com maior visibilidade pelas organizações de saúde nacionais e mundiais (10). Após a Declaração de Alma-Ata, em 1978, diversas políticas de atenção à saúde emergiram nas Américas, baseadas no novo conceito de APS, orientadas para a cobertura universal em saúde com acesso para toda a população $(1,6,15)$.

A partir da década de 1990, como resultado de tensões culturais em diversas regiões do continente, advindas do rápido processo de globalização, teve início uma discussão sobre as condições sanitárias e de saúde dos povos indígenas (10). Em 1993, a Organização Pan-Americana da Saúde/Organização Mundial da Saúde (OPAS/OMS) instituiu a Iniciativa de Salud de los Pueblos Indígenas, que apoiava: 1) a necessidade de considerar a saúde com um critério integral; 2) o direito dos povos indígenas à autodeterminação e à participação sistemática; 3) o respeito pela cultura indígena e sua revitalização e 4) a reciprocidade nas relações com outros grupos (7). De 2006 e 2011, a OPAS/OMS propôs a incorporação da perspectiva indígena na elaboração de políticas nacionais de saúde, permitindo a integração entre medicina tradicional indígena e medicina científica ocidental. Foi proposta ainda a inclusão do termo "medicina intercultural" às políticas de saúde. Dessa forma, é possível formar alianças estratégicas na APS, respeitando recursos e tratamentos da medicina indígena como via alternativa e compreendendo a saúde do indivíduo de forma holista, determinada por fatores sociais, econômicos e culturais $(15,16)$. A maioria dos países sul-americanos possui, atualmente, ministérios e órgãos específicos para manejo da saúde indígena. No entanto, em muitas regiões não existe apoio político consistente, tornando as políticas de saúde ineficazes para esses povos (10).

Entre os principais obstáculos no acesso à APS enfrentados pelos povos indígenas sul-americanos, conforme os artigos pesquisados, estão a dificuldade de acesso longitudinal aos serviços de saúde mais próximos das aldeias; linguagem e ilustrações das cartilhas de educação em saúde inapropriadas ao contexto indígena; dificuldade de comunicação com os profissionais de saúde; carência de meios de transporte adequados até as unidades de saúde; escassez de dados epidemiológicos das aldeias indígenas; ausência de informação sobre as culturas indígenas locais; e medo de discriminação ou humilhação por parte do paciente indígena $(3,17,18)$.

Foram limitações do presente trabalho a não inclusão de documentos governamentais ou de outras fontes sobre políticas específicas, já que a produção de artigos científicos sobre o tema tratado pode necessitar de tempo considerável para refletir mudanças, e a inclusão somente de artigos de acesso aberto, o que pode ter resultado na exclusão de estudos importantes de acesso limitado aos autores. Além disso, como limitações significativas pode-se citar o curto espaço temporal de análise retrospectiva (10 anos) e a carência de artigos científicos sobre saúde indígena, que se reflete em dados escassos e pouco precisos, em termos antropológicos e epidemiológicos. Apesar dessas limitações, este trabalho oferece uma importante contribuição, apresentando o atual panorama da saúde indígena na América do Sul e o déficit em pesquisas sobre saúde indígena em todo o continente, comprometendo uma análise mais profunda do cenário sul-americano. A ausência de panoramas de saúde em alguns países (Guiana, Uruguai, Venezuela, Suriname) ou falta de detalhamento apropriado em outros (Bolívia, Equador, Paraguai) demonstra a escassez de estudos nessas comunidades. Dessa forma, ainda são imprescindíveis estudos que investiguem de modo mais profundo e detalhado o panorama da saúde dos indígenas sul-americanos.

Apesar da escassez de informações, foi possível concluir que os grupos indígenas da América do Sul, apesar das diferenças culturais e assistenciais de cada país, enfrentam enfermidades semelhantes, como doenças infecto-parasitárias, coexistindo com moléstias da modernidade, como etilismo e doenças crônicas não transmissíveis. Frente a tais diferenças culturais e aos obstáculos para o acesso à saúde nas aldeias, a APS não é estabelecida de forma plena, eficaz e longitudinal. Apesar dos esforços das políticas públicas sul-americanas relativas ao cuidado com a população indígena, os avanços esperados não são completamente visíveis pelos registros da literatura e a maioria das aldeias enfrenta os mesmos problemas de quando os trabalhos começaram a surgir, como dificuldade de acesso às localidades, alta rotatividade de profissionais e dissonância entre cuidados biomédicos e saberes tradicionais.

Mais especificamente, a falta de acesso geográfico aos serviços de saúde foi o principal problema enfrentado pelos povos indígenas do Peru. Na Bolívia e no Brasil, a falta de comunicação adequada entre profissionais de saúde e pacientes indígenas dificulta a adesão desses povos aos serviços formais de saúde. $\mathrm{Na}$ Colômbia, o direito à saúde dos povos indígenas não é assegurado pelo governo, o que se reflete em altas taxas de mortalidade ainda presentes nas tribos. No Chile, a falta de informação e educação sobre a cultura e crenças dos indígenas inviabiliza o atendimento adequado a essa população.

Apesar das perspectivas e políticas com objetivo de melhorar a APS dos povos indígenas, ainda há muitas barreiras 
que necessitam de estratégias específicas e investimentos voltados para a saúde desses grupos. A expansão do cuidado biomédico e os esforços para alcançar as aldeias indígenas precisam estar estrategicamente aliados ao conhecimento sobre tais povos, de forma a realmente acolher a saúde indigenista e suas particularidades.

Conflitos de interesse. Nada declarado pelos autores.

\section{REFERÊNCIAS}

1. Díaz A, Arana A, Vargas-Machuca R, Antiporta D. Situación de salud y nutrición de niños indígenas y niños no indígenas de la Amazonia peruana. Rev Panam Salud Publica. 2015;38(1):49-56.

2. Valeggia C. The global and the local: health in Latin American indigenous women. Health Care Women Int. 2016;37(4):463-77.

3. Castro A, Savage V, Kaufman H. Assessing equitable care for Indigenous and Afrodescendant women in Latin America. Rev Panam Salud Publica. 2015;38(2):96-109.

4. Walker RS, Sattenspiel L, Hill KR. Mortality from contact-related epidemics among indigenous populations in Greater Amazonia. Sci Rep. 2015;5:1-9.

5. Agudelo-Suárez AA, Martínez-Herrera E, Posada-López A, Rocha-Buelvas A. Ethnicity and health in Colombia: what do self-perceived health indicators tell us? Ethn Dis. 2016;26(2):147-56.

6. World Health Organization. International Conference on Primary Health Care, AlmaAta. Alma-Ata; 1978. Disponível em: www. who.int/publications/almaata_declaration_en.pdf?ua=1\&ua=1 Acessado em 8 de agosto de 2018

7. Brasil. Lei 8080/1990. Disponível em: http:/ / www.planalto.gov.br/ccivil_03/ leis /L8080.htm Acessado em 27 de dezembro de 2017.

8. Brasil. Lei 9836/1990. Disponível em: http:/ / www.planalto.gov.br/ccivil_03/ leis/L9836.htm\#art1 Acessado em 27 de dezembro de 2017.

9. Benevides L, Benevides LAC, do Nascimento WF. A atenção à saúde dos povos indígenas do Brasil: das missões ao subsistema. Tempus Actas Saude Colet. 2014;8(1):29-39.

10. Nureña CR. Incorporación del enfoque intercultural en el sistema de salud peruano: la atención del parto vertical. Rev Panam Salud Publica. 2009;26(4):368-76.

11. Pereira AMM, de Castro ALB, Oviedo RAM, Barbosa LG, Gerassi CD, Giovanella L. Atenção primária à saúde na América do Sul em perspectiva comparada: mudanças e tendências. Saude Debate. 2012; 36(94):482-99.

12. Silveira NH. Políticas públicas de saúde e indigenismo na América Latina. Estud Iberoam. 2017;43(1):135-8.

13. Mendes KS, Silveira RCCP, Galvão CM. Revisão integrativa: método de pesquisa para a incorporação de evidências na saúde e na enfermagem. Texto Contexto Enferm. 2008;17(4):758-64.

14. Melnyk BM, Fineout-Overholt E. Making the case for evidence-based practice. Em: Melnyk BM, Fineout-Overholt E. Evidencebased practice in nursing \& healthcare: a guide to best practice. Filadélfia: Lippincott Williams \& Wilkins; 2005. Pp. 3-24.

15. Patiño Suaza AE, Sandín Vásquez M. Dialogo y respeto: bases para la construcción de un sistema de salud intercultural para las comunidades indígenas de Puerto Nariño, Amazonas, Colombia. Salud Colectiva. 2014;10(3):379-96.

16. Knipper M. Más allá de lo indígena: salud e interculturalidad a nivel global. Rev Peru Med Exp Salud Publica. 2010;27(1):94-101.

17. Gianella C, Ugarte-Gil C, Caro G, Aylas R, Castro C, Lema C. TB in vulnerable populations: the case of an indigenous community in the Peruvian Amazon. Health Hum Rights. 2016;18(1):55-68.

18. Pérez C, Nazar G, Cova F. Facilitadores y obstaculizadores de la implementación de la política de salud intercultural en Chile. Rev Panam Salud Publica. 2016;39(2): 122-7.

19. Yajahuanca RA, Diniz CSG, Cabral CdS. É preciso "ikarar os kutipados": interculturalidade e assistência à saúde na Amazônia peruana. Cienc Saude Coletiva. 2015;20(9): 2837-46.

20. Brierley CK, Suarez N, Arora G, Graham D. Healthcare access and health beliefs of the indigenous peoples in remote Amazonian Peru. Am J Trop Med Hyg. 2014;90(1): 180-3.

21. Undurragaa EA, Nica V, Zhang R, Mensah IC, Godoy RA. Individual health and the visibility of village economic inequality: Longitudinal evidence from native Amazonians in Bolivia. Econ Hum Biol. 2016;23:18-26.

22. Dell'Arciprete A, Braunstein I, Touris C, Dinardi G, Llovet I, Sosa-Estani S. Cultural barriers to effective communication between Indigenous communities and health care providers in Northern Argentina: an anthropological contribution to Chagas disease prevention and control. Int J Equity Health. 2014;13(6):1-10.

23. Hita SR. Aspectos interculturales de la reforma del sistema de salud en Bolivia. Rev Peru Med Exp Salud Publica. 2014;31(4): 762-68.

24. Mirassou CS. Sistema de salud pública y comunidades indígenas de la provincia de Formosa. Medicina (B Aires). 2013;73: 453-6.

25. López-Cevallos D, Chi C, Ortega F. Consideraciones para la transformación del sistema de salud del Ecuador desde una perspectiva de equidad. Rev Salud Publica. 2014;16(3):346-59.

26. Coates AR, Marchito SdP, Vitoy B. Indigenous child health in Brazil: the evaluation of impacts as a human rights issue. Health Hum Rights. 2016;18(1):221-34.
Declaração. As opiniões expressas no manuscrito são de responsabilidade exclusiva dos autores e não refletem necessariamente a opinião ou políticas da RPSP/PAJPH ou da Organização PanAmericana da Saúde (OPAS).

27. Santos MM, Cruz KJC, de Sá LCR, Batista CC, Aguiar EMG, Nogueira AMT. Assistência prestada pelo Sistema Único de Saúde de Teresina à população indígena do Maranhão, 2011: um estudo descritivo. Epidemiol Serv Saude. 2016;25(1):127-36.

28. Coelho V, Shankland A. Making the right to health a reality for Brazil's indigenous peoples: innovation, decentralization and equity. MEDICC Review. 2011;13(3):50-3.

29. Diehl EE, Langdon EJ. Transformações na atenção à saúde indígena: tensões e negociações em um contexto indígena brasileiro. Univ Humanist. 2015;(80):213-36

30. Silva RP, Barcelos AC, Hirano BQL Izzo RS, Calafate JMS, Soares TO. A experiência de alunos do PET-Saúde com a saúde indígena e o programa Mais Médicos. Interface (Botucatu). 2015;19(1):1005-14.

31. Cardoso MD. Saúde e povos indígenas no Brasil: notas sobre alguns temas equívocos na política atual. Cad Saude Publica. 2014;30(4):860-6.

32. Taveira ZZ, Scherer MDA, Diehl EE. Implantação da telessaúde na atenção à saúde indígena no Brasil. Cad Saude Publica. 2014;30(8):1793-7.

33. Fontão MAB, Pereira EL. Projeto Mais Médicos na saúde indígena: reflexões a partir de uma pesquisa de opinião. Interface (Botucatu). 2017;21(1):1169-80.

34. Diehl EE, Langdon EJ, Dias-Scopel RP. Contribuição dos agentes indígenas de saúde na atenção diferenciada à saúde dos povos indígenas brasileiros. Cad Saude Publica. 2012;28(5):819-31.

35. Borghi AC, Alvarez AM, Marcon SS, Carreira L. Singularidades culturais: o acesso do idoso indígena aos serviços públicos de saúde. Rev Esc Enferm USP. 2015;49(4):589-95.

36. Ribeiro AA, Aciole GG, Arantes CIS, Reading J, Kurtz DLM, Rossi LA. Processo de trabalho e produção do cuidado em um serviço de saúde indígena no Brasil. Esc Anna Nery. 2017;21(4):1-9.

37. de Moura-Pontes AL, Garnelo L. La formación y el trabajo del agente indígena de salud en el Subsistema de Salud Indígena en Brasil. Salud Publica Mex. 2014;56(4): 386-92.

38. Scopel D, Dias-Scopel RP, Langdon EJ. Intermedicalidade e protagonismo: a atuação dos agentes indígenas de saúde Munduruku da Terra Indígena KwatáLaranjal, Amazonas, Brasil. Cad Saude Publica. 2015;31(12):2559-68.

39. Diehl EE, Pellegrini MA. Saúde e povos indígenas no Brasil: o desafio da formação e educação permanente de trabalhadores para atuação em contextos interculturais. Cad Saude Publica. 2014; 30(4):867-74. 
40. Pontes ALM, Rego S, Garnelo L. O modelo de atenção diferenciada nos Distritos Sanitários Especiais Indígenas: reflexões a partir do Alto Rio Negro/AM, Brasil. Cienc Saude Coletiva. 2015;20(10):3199-210.

41. Aguirre S, Cuellar CM, Herrero MB, Cortesi GC, Romero NG, Alvarez M, et al. Prevalence of tuberculosis respiratory symptoms and associated factors in the indigenous populations of Paraguay (2012). Mem Inst Oswaldo Cruz. 2017;112(7): 474-84.

42. Novo MP. Política e intermedicalidade no Alto Xingu: do modelo à prática de atenção à saúde indígena. Cad Saude Publica. 2011;27(7):1362-70.
43. Pena JL, Heller L. Saneamento e saúde indígena: uma avaliação na população Xakriabá, Minas Gerais. Eng Sanit Ambiente. 2008;13(1):63-72.

44. Rissardo LK, Carreira L. Organização do serviço de saúde e cuidado ao idoso indígena: sinergias e singularidades do contexto profissional. Rev Esc Enferm USP. 2014;48(1):73-81.

45. Rissardo LK, Titonelli Alvim NA, Marcon SS, Carreira L. Práticas de cuidado ao idoso indígena-atuação dos profissionais de saúde. Rev Bras Enferm. 2014;67(6):920-7.

46. Sousa MDC, Scatena JHG, Santos RVO. Sistema de Informação da Atenção à Saúde Indígena (SIASI): criação, estrutura e funcionamento. Cad Saude Publica. 2007;23(4):853-61.

47. Pontes ALM, Garnelo L, Rego S. Reflexões sobre questões morais na relação de indígenas com os serviços de saúde. Rev Bioet. 2014;22(2):337-46.

48. Gil LP. Políticas de saúde, pluralidade terapêutica e identidade na Amazônia. Saude Soc. 2007;16(2):48-60.

Manuscrito recebido em 12 de janeiro de 2018. Aceito em versão revisada em 22 de agosto de 2018.
ABSTRACT

Primary health care for South-American indigenous peoples: an integrative review of the literature
Objective. To review the literature regarding the access to primary health care (PHC) by indigenous communities in South America, identifying the main access barriers.

Method. Integrative review of articles published from 2007 to 2017 in the LILACS, PubMed, and SciELO databases. The search terms "indigenous AND health AND Brazil" and "indigenous AND health NOT Brazil" were used in Portuguese and English. Articles published in English, Portuguese, or Spanish, focusing strictly on $\mathrm{PHC}$ and on South-American indigenous populations were included.

Results. Forty articles describing aspects of PHC for indigenous populations in eight countries - Brazil, Peru, Colombia, Bolivia, Argentina, Chile, Paraguay, and Ecuador - were included. The main barriers to accessing PHC were the difficulty of reaching the health care facilities closest to villages; difficulty in communicating with health care professionals; inadequate transportation to the health care units; lack of epidemiological data on indigenous villages; lack of information regarding local indigenous cultures; and fear of discrimination or humiliation on the part of indigenous patients. Conclusions. Studies regarding the health of indigenous populations in South America are scarce. It is evident that national health systems still need to advance towards an intercultural medicine that respects the social, cultural, and economic realities of all communities, with knowledge and consideration for different forms of care. 
RESUMEN

Atención primaria en salud a indígenas de América del Sur: revisión integrativa de la bibliografía

Objetivo. Revisar la bibliografía acerca del acceso a la atención primaria de salud (APS) de las comunidades indígenas de América del Sur, e identificar los principales obstáculos a ese acceso.

Métodos. Revisión integrativa de artículos publicados desde 2007 a 2017 en las bases de datos LILACS, PubMed y SciELO. Para la búsqueda se utilizaron los siguientes descriptores: "indígenas AND salud AND Brasil" e "indígenas AND salud NOT Brasil" en portugués e inglés. Se incluyeron artículos publicados en inglés, portugués y español que abordaran estrictamente la APS en indígenas de América del Sur.

Resultados. Se incluyeron 40 artículos que describieron los aspectos de la APS en indígenas de ocho países: Argentina, Bolivia, Chile, Colombia, Brasil, Ecuador, Paraguay y Perú. Los principales obstáculos de acceso a la APS detectados fueron la dificultad de acceso a los servicios de salud más próximos de las aldeas; lenguaje e ilustraciones de las cartillas de educación en salud inapropiadas al contexto indígena; dificultad de comunicación con los profesionales de salud; carencia de medios de transporte adecuados hasta las unidades de salud; escasez de datos epidemiológicos de las aldeas indígenas; ausencia de información sobre las culturas indígenas locales; y miedo de discriminación o humillación en el paciente indígena.

Conclusiones. Aún son escasos los estudios sobre la salud indígena en América del Sur. Es evidente que los sistemas de salud nacionales aún necesitan avanzar hacia una medicina intercultural, con respeto a las realidades sociales, culturales y económicas de todas las comunidades asistidas, con conocimiento y consideración de las diferentes formas de cuidados.

Palabras clave Poblaciones indígenas; salud de poblaciones indígenas; atención primaria de salud; América Latina. 